\title{
ABOUT THE LINEAR-QUADRATIC REGULATOR PROBLEM UNDER A FRACTIONAL BROWNIAN PERTURBATION*,**
}

\author{
M.L. Kleptsyna ${ }^{1}$, Alain Le Breton ${ }^{2}$ and M. Viot ${ }^{2}$
}

\begin{abstract}
In this paper we solve the basic fractional analogue of the classical linear-quadratic Gaussian regulator problem in continuous time. For a completely observable controlled linear system driven by a fractional Brownian motion, we describe explicitely the optimal control policy which minimizes a quadratic performance criterion.
\end{abstract}

Mathematics Subject Classification. 93E20, 60G15, 60G44.

Received September 12, 2002.

\section{INTRODUCTION}

Several contributions in the literature have been already devoted to the extension of the classical theory of continuous-time stochastic systems driven by Brownian motions to analogues in which the driving processes are fractional Brownian motions (fBm's for short). The tractability of the standard problems in prediction, parameter estimation and filtering is now rather well understood (see, e.g. $[4,6-8,11,12]$ and references therein). Concerning optimal control problems, as far as we know, it is far from fully demonstrated (nevertheless, see [5] for an attempt in a general setting and [9] for a study in an elementary archetypal model). Here our aim is to illustrate the actual solvability of control problems by exhibiting an explicit solution for the case of the simplest linear-quadratic model.

We deal with the fractional analogue of the so-called linear-quadratic Gaussian regulator problem in one dimension. The real-valued state process $X=\left(X_{t}, t \in[0, T]\right)$ is governed by the stochastic differential equation

$$
\mathrm{d} X_{t}=a(t) X_{t} \mathrm{~d} t+b(t) u_{t} \mathrm{~d} t+c(t) \mathrm{d} B_{t}^{H}, t \in[0, T], X_{0}=x
$$

which is as usual interpreted as an integral equation. Here $x$ is a fixed initial condition, $B^{H}=\left(B_{t}^{H}, t \in[0, T]\right)$ is a normalized $\mathrm{fBm}$ with the Hurst parameter $H$ in $[1 / 2,1)$ and the coefficients $a=(a(t), t \in[0, T]), b=(b(t)$, $t \in[0, T])$ and $c=(c(t), t \in[0, T])$ are fixed (deterministic) continuous functions. We suppose that $X$ is

Keywords and phrases: Fractional Brownian motion, linear system, optimal control, quadratic payoff.

* M.L. Kleptsyna's research was supported by the Grants RFBR 0001-00571 69 0015-96116, INTAS 99-00559 and CNRS 00-01-22000a. She is presently with Laboratoire de Statistique et Processus, Université du Maine, avenue Olivier Messiaen, 72085 Le Mans Cedex 9, France.

** Le Breton's research was supported by the Project IDOPT, CNRS-UJF-INPG-INRIA.

1 Institute of Information Transmission Problems, Bolshoi Karetnii Per. 19, Moscow 101475, Russia;

e-mail: Marina.Kleptsyna@univ-lemans.fr

2 Laboratoire de Modélisation et Calcul, Université J. Fourier, BP. 53, 38041 Grenoble Cedex 9, France;

e-mail: Alain.Le-Breton@imag.fr, A-C.Fouilhe-Viot@wanadoo.fr 
completely observed and that a closed-loop control of the system is available in the sense that at each time $t \in[0, T]$ one may choose the input $u_{t}$ in view of the passed observations $\left\{X_{s}, s \leq t\right\}$ in order to drive the corresponding state, $X_{t}=X_{t}^{u}$ say. Then, given a cost function which evaluates the performance of the control actions, the classical problem of controlling the system dynamics on the time interval $[0, T]$ so as to minimize this cost occurs. Here we consider the quadratic payoff $J$ defined for a control policy $u=\left(u_{t}, t \in[0, T]\right)$ by

$$
J(u)=\mathbb{E}\left\{q_{T} X_{T}^{2}+\int_{0}^{T}\left[q(t) X_{t}^{2}+r(t) u_{t}^{2}\right] \mathrm{d} t\right\},
$$

where $q_{T}$ is a positive constant and $q=(q(t), t \in[0, T])$ and $r=(r(t), t \in[0, T])$ are fixed (deterministic) positive continuous functions. It is well-known that when $H=1 / 2$ and hence the noise in (0.1) is a Brownian motion, then (see, e.g. $[1,10]$ ), the solution $\bar{u}$ to the corresponding problem, called the optimal control, is provided for all $t \in[0, T]$ by the instantaneous linear feedback

$$
\bar{u}_{t}=-\frac{b(t)}{r(t)} \pi(t) \bar{X}_{t} ; \quad \bar{X}_{t}=X_{t}^{\bar{u}}
$$

where $\pi=(\pi(t), t \in[0, T])$ is the unique nonnegative solution of the backward Riccati differential equation

$$
\dot{\pi}(t)=-2 a(t) \pi(t)-q(t)+\frac{b^{2}(t)}{r(t)} \pi^{2}(t) ; \quad \pi(T)=q_{T} .
$$

Moreover the optimal cost $J(\bar{u})$ is given by

$$
J(\bar{u})=\pi(0) x^{2}+\int_{0}^{T} \pi(t) c^{2}(t) \mathrm{d} t .
$$

Our main goal here is to show that actually when the system $(0.1)$ is driven by a fBm with some $H \in(1 / 2,1)$ instead of a Brownian motion, an explicit solution to the optimal control problem under the performance criterion (0.2) is still available.

The paper is organized as follows. At first in Section 1, we fix some notations and preliminaries. Then, in Section 3, the concerned closed-loop control problem is solved: the optimal control is defined as a linear but not instantaneous feedback which involves the solution of a Volterra type integral equation; moreover a representation of the optimal cost is also provided. Globally, the solution of the problem is expressed in terms of the solutions of a family of two-dimensional backward differential Riccati equations which for $H=1 / 2$ actually all reduce to the standard one-dimensional Riccati equation (0.4).

\section{Preliminaries}

In what follows all random variables and processes are defined on a given stochastic basis $(\Omega, \mathcal{F}, \mathbb{P})$. Moreover the natural filtration of a process is understood as the $\mathbb{P}$-completion of the filtration generated by this process. Here, for some fixed $T>0$ and $H \in[1 / 2,1), B^{H}=\left(B_{t}^{H}, t \in[0, T]\right)$ is a normalized fractional Brownian motion on $[0, T]$ with Hurst parameter means that $B^{H}$ is a Gaussian process with continuous paths such that $B_{0}^{H}=0$, $\mathbb{E} B_{t}^{H}=0$ and

$$
\mathbb{E} B_{s}^{H} B_{t}^{H}=\frac{1}{2}\left[s^{2 H}+t^{2 H}-|s-t|^{2 H}\right], 0 \leq s, t \leq T
$$

Of course the fBm reduces to the standard Brownian motion when $H=1 / 2$. For $H \neq 1 / 2$, the fBm is outside the world of semimartingales but a theory of stochastic integration w.r. to fBm has been developed (see, e.g. $[2,3])$. 
Actually the case of deterministic integrands, which is sufficient for the purpose of the present paper, is easy to handle (see, e.g. [11]).

- Fundamental martingale associated to $B^{H}$ : there are simple integral transformations which change the fBm to martingales (see $[8,11,12]$ ). In particular, defining for $0<s<t \leq T$

$$
\begin{gathered}
k_{H}(t, s)=\kappa_{H}^{-1} s^{\frac{1}{2}-H}(t-s)^{\frac{1}{2}-H} ; \kappa_{H}=2 H \Gamma(3 / 2-H) \Gamma(H+1 / 2), \\
w_{t}^{H}=\lambda_{H}^{-1} t^{2-2 H} ; \quad \lambda_{H}=\frac{2 H \Gamma(3-2 H) \Gamma(H+1 / 2)}{\Gamma(3 / 2-H)}, \\
M_{t}^{H}=\int_{0}^{t} k_{H}(t, s) \mathrm{d} B_{s}^{H},
\end{gathered}
$$

then the process $M^{H}$ is a Gaussian martingale, called in [11] the fundamental martingale, whose variance function $\left\langle M^{H}\right\rangle$ is nothing but the function $w^{H}$. Actually, the natural filtration of $M^{H}$ coincides with the natural filtration $\left(\mathcal{F}_{t}^{H}\right)$ of $B^{H}$. In particular, we have the direct consequence of the results of [8] that, given a suitably regular deterministic function $c=(c(t), t \in[0, T])$, the following representation holds:

$$
\int_{0}^{t} c(s) \mathrm{d} B_{s}^{H}=\int_{0}^{t} K_{H}^{c}(t, s) \mathrm{d} M_{s}^{H}
$$

where for $H \in(1 / 2,1)$ the function $K_{H}^{c}$ is given by

$$
K_{H}^{c}(t, s)=H(2 H-1) \int_{s}^{t} c(r) r^{H-\frac{1}{2}}(r-s)^{H-\frac{3}{2}} \mathrm{~d} r, 0 \leq s \leq t,
$$

and for $H=1 / 2$ the convention $K_{1 / 2}^{c}(t,.) \equiv c$ for all $t$ is used. Conversely, given a suitably regular deterministic function $f=(f(t), t \in[0, T])$, we have also:

$$
\int_{0}^{t} f(s) \mathrm{d} M_{s}^{H}=\int_{0}^{t} k_{H}^{f}(t, s) \mathrm{d} B_{s}^{H}
$$

where the function $k_{H}^{f}$ is given by

$$
k_{H}^{f}(t, s)=-\kappa_{H}^{-1} s^{\frac{1}{2}-H} \frac{\mathrm{d}}{\mathrm{d} s} \int_{s}^{t}(r-s)^{\frac{1}{2}-H} f(r) \mathrm{d} r .
$$

- Admissible controls: let $\mathcal{U}_{H}$ the class of $\left(\mathcal{F}_{t}^{H}\right)$-adapted processes $u=\left(u_{t}, t \in[0, T]\right)$ such that the stochastic differential equation (0.1) has a unique strong solution $X^{u}$ which satisfies $J(u)<+\infty$, where $J(u)$ is evaluated according to $(0.2)$ with $X=X^{u}$. Of course then $X^{u}$ is a $\left(\mathcal{F}_{t}^{H}\right)$-adapted process. Actually, as mentioned in the Introduction, for control purpose we are interested in closed-loop policies. So, we introduce the class of admissible controls as the class $\mathcal{U}_{\text {ad }}$ of those $u$ 's in $\mathcal{U}_{H}$ which are $\left(\mathcal{F}_{t}^{u}\right)$-adapted processes where $\left(\mathcal{F}_{t}^{u}\right)$ is the natural filtration of the corresponding state process $X^{u}$. For $u \in \mathcal{U}_{\mathrm{ad}}$, the pair $\left(u, X^{u}\right)$ is called an admissible pair and if $\bar{u} \in \mathcal{U}_{\mathrm{ad}}$ is such that

$$
J(\bar{u})=\inf \left\{J(u), u \in \mathcal{U}_{\mathrm{ad}}\right\}
$$

then it is called an optimal control and $(\bar{u}, \bar{X})$, where $\bar{X}=X^{\bar{u}}$, is called an optimal pair and the quantity $J(\bar{u})$ is called the optimal cost. 


\section{Solution of the optimal CONTROL PROBlem}

In order to state our main result, starting from the solution $\pi$ of the Riccati equation (0.4), for any fixed $s \in[0, T]$, we introduce auxiliary deterministic functions $\gamma(., s)=(\gamma(t, s), t \in[s, T])$ and $\lambda(., s)=(\lambda(t, s)$, $t \in[s, T])$. They are the solutions of the following backward differential equations in the variable $t$ on $[s, T]$ :

$$
\begin{aligned}
& \dot{\gamma}(., s)=-a\left[\gamma(., s)+K_{H}^{c}(., s) \pi\right]-q K_{H}^{c}(., s)+\frac{b^{2}}{r} \pi \gamma(., s) ; \quad \gamma(T, s)=q_{T} K_{H}^{c}(T, s), \\
& \dot{\lambda}(., s)=-2 a K_{H}^{c}(., s) \gamma(., s)-q\left[K_{H}^{c}(., s)\right]^{2}+\frac{b^{2}}{r} \gamma^{2}(., s) ; \quad \lambda(T, s)=q_{T}\left[K_{H}^{c}(T, s)\right]^{2} .
\end{aligned}
$$

Let us mention that actually the $2 \times 2$ matrix-valued function $\Gamma(., s)=(\Gamma(t, s), t \in[s, T])$ where

$$
\Gamma(t, s)=\left(\begin{array}{cc}
\pi(t) & \gamma(t, s) \\
\gamma(t, s) & \lambda(t, s)
\end{array}\right),
$$

is the unique nonnegative symmetric solution of the backward Riccati equation

$$
\begin{aligned}
\dot{\Gamma}(., s)= & -a\left\{\mathcal{K}_{H}^{c}(., s) e_{1}^{\prime} \Gamma(., s)+\Gamma(., s) e_{1}\left[\mathcal{K}_{H}^{c}(., s)\right]^{\prime}\right\} \\
& -q \mathcal{K}_{H}^{c}(., s)\left[\mathcal{K}_{H}^{c}(., s)\right]^{\prime}+\frac{b^{2}}{r} \Gamma(., s) e_{1} e_{1}^{\prime} \Gamma(., s) ; \Gamma(T, s)=q_{T} \mathcal{K}_{H}^{c}(T, s)\left[\mathcal{K}_{H}^{c}(T, s)\right]^{\prime},
\end{aligned}
$$

with the vectors $e_{1}$ and $\mathcal{K}_{H}^{c}(t, s)$ in $\mathbb{R}^{2}$ given by

$$
e_{1}=\left(\begin{array}{l}
1 \\
0
\end{array}\right) ; \quad \mathcal{K}_{H}^{c}(t, s)=\left(\begin{array}{c}
1 \\
K_{H}^{c}(t, s)
\end{array}\right) .
$$

We shall use the following notations:

$$
\bar{\gamma}(s)=\gamma(s, s) ; \quad \bar{\lambda}(s)=\lambda(s, s),
$$

where $\gamma(., s)$ and $\lambda(., s)$ are defined by equations (2.1) and (2.2), respectively. We introduce also the function $\bar{k}(t, s)=k_{H}^{\bar{\gamma}}(t, s)$ by substituting $\bar{\gamma}$ for $f$ in the definition (1.8), i.e.:

$$
\bar{k}(t, s)=-\kappa_{H}^{-1} s^{\frac{1}{2}-H} \frac{\mathrm{d}}{\mathrm{d} s} \int_{s}^{t}(r-s)^{\frac{1}{2}-H} \bar{\gamma}(r) \mathrm{d} r .
$$

Theorem 2.1. There exists a unique opimal control $\bar{u}$ in $\mathcal{U}_{\mathrm{ad}}$ and the optimal pair $(\bar{u}, \bar{X})$ is governed on $[0, T]$ by the system

$$
\begin{gathered}
\bar{u}_{t}=-\frac{b(t)}{r(t)}\left[\pi(t) \bar{X}_{t}+\bar{v}_{t}\right] ; \quad \bar{X}_{t}=X_{t}^{\bar{u}} \\
\bar{v}_{t}=\int_{0}^{t}\left[-a(s)+\frac{b^{2}(s)}{r(s)} \pi(s)\right] \bar{v}_{s} \mathrm{~d} s+\int_{0}^{t}\left[\frac{\bar{k}(t, s)}{c(s)}-\pi(s)\right]\left\{\mathrm{d} \bar{X}_{s}-\left[a(s) \bar{X}_{s}+b(s) \bar{u}_{s}\right] \mathrm{d} s\right\},
\end{gathered}
$$

where $\pi$ and $\bar{k}$ are defined by (0.4) and (2.4), respectively. Moreover the optimal cost is given by

$$
J(\bar{u})=\pi(0) x^{2}+\int_{0}^{T} \bar{\lambda}(t) \mathrm{d} w_{t}^{H},
$$

where $\bar{\lambda}$ is defined by (2.3). 
Remark 2.2. (a) Observe that in the case $H=1 / 2$, for all $0 \leq s \leq t \leq T$, the entries $\gamma(t, s), \lambda(t, s)$ and $\bar{k}(t, s)$ reduce to $\pi(t) c(s), \pi(t) c^{2}(s)$ and $\pi(s) c(s)$, respectively. Hence, it is readily seen that $\bar{v} \equiv 0, \bar{u}=-(b / r) \pi \bar{X}$ and $\bar{\lambda}(t) \mathrm{d} w_{t}^{H}=\pi(t) c^{2}(t) \mathrm{d} t$. So, finally, the statement in Theorem 2.1 reduces to the well-known result recalled in the Introduction.

(b) It is worth to mention that actually the additional term $\bar{v}_{t}$ which appears in the case $H>1 / 2$ can be interpreted in terms of the predictors at time $t$ of the noise component $\widetilde{B}_{\tau}=\int_{0}^{\tau} c(s) \mathrm{d} B_{s}^{H}, t \leq \tau \leq T$ based on the observed optimal dynamics $\left(\bar{X}_{s}, s \leq t\right)$ up to time $t$. Precisely, one can rewrite

$$
\bar{v}_{t}=\int_{t}^{T} \phi(\tau, t) \pi(\tau) \mathrm{d} \hat{B}_{\tau}^{t},
$$

where $\hat{B}_{\tau}^{t}=\mathbb{E}\left(\widetilde{B}_{\tau} / \mathcal{F}_{t}^{\bar{u}}\right)$ and

$$
\phi(\tau, t)=\exp \left\{\int_{t}^{\tau}\left[a(u)-\frac{b^{2}(u)}{r(u)} \pi(u)\right] \mathrm{d} u\right\} .
$$

This will be made clear in Remark 2.5 after the proof of Theorem 2.1.

Before turning to the proof of Theorem 2.1, at first we derive a sufficient condition for optimality in $\mathcal{U}_{H}$. Given $u \in \mathcal{U}_{H}$ and $X=X^{u}$, we introduce the following backward stochastic differential equation in the pair of unknown $\left(\mathcal{F}_{t}^{H}\right)$-adapted processes $p=\left(p_{t}, t \in[0, T]\right)$ and $\beta=\left(\beta_{t}, t \in[0, T]\right)$ :

$$
\mathrm{d} p_{t}=-a(t) p_{t} \mathrm{~d} t-q(t) X_{t} \mathrm{~d} t+\beta_{t} \mathrm{~d} M_{t}^{H}, t \in[0, T] ; p_{T}=q_{T} X_{T} .
$$

Lemma 2.3. Suppose that $u \in \mathcal{U}_{H}$ is such that $u=-(b / r) p$ where $(p, \beta)$ is a pair of $\left(\mathcal{F}_{t}^{H}\right)$-adapted processes which satisfies equation (2.10). Then u minimizes $J$ over $\mathcal{U}_{H}$.

Proof. Given an arbitrary $u^{*} \in \mathcal{U}_{H}$, for which we use the notation $X_{t}^{*}=X_{t}^{u^{*}}$, we evaluate the difference $J\left(u^{*}\right)-J(u)$ between the corresponding cost and the cost for the announced candidate $u$ to optimality over $\mathcal{U}_{H}$. Of course, we can write

$$
J\left(u^{*}\right)-J(u)=\mathbb{E}\left\{q_{T}\left[\left(X_{T}^{*}\right)^{2}-X_{T}^{2}\right]+\int_{0}^{T}\left\{q(t)\left[\left(X_{t}^{*}\right)^{2}-X_{t}^{2}\right]+r(t)\left[\left(u_{t}^{*}\right)^{2}-u_{t}^{2}\right]\right\} \mathrm{d} t\right\} .
$$

Using the equality $\left(y^{*}\right)^{2}-y^{2}=\left(y^{*}-y\right)^{2}+2 y\left(y^{*}-y\right)$ and exploiting the property $u=-(b / r) p$, it is readily seen that

$$
J\left(u^{*}\right)-J(u)=\Delta_{1}+2 \Delta_{2},
$$

where

$$
\begin{aligned}
& \Delta_{1}=\mathbb{E}\left\{q_{T}\left[X_{T}^{*}-X_{T}\right]^{2}+\int_{0}^{T}\left\{q(t)\left[X_{t}^{*}-X_{t}\right]^{2}+r(t)\left[u_{t}^{*}-u_{t}\right]^{2}\right\} \mathrm{d} t\right\}, \\
& \Delta_{2}=\mathbb{E}\left\{q_{T} X_{T}\left[X_{T}^{*}-X_{T}\right]+\int_{0}^{T}\left\{q(t) X_{t}\left[X_{t}^{*}-X_{t}\right]-b(t) p_{t}\left[u_{t}^{*}-u_{t}\right]\right\} \mathrm{d} t\right\} .
\end{aligned}
$$

But, rewriting the quantity in the last integral as

$$
\left(X_{t}^{*}-X_{t}\right)\left[q(t) X_{t}+a(t) p_{t}\right]-p_{t}\left[a(t)\left(X_{t}^{*}-X_{t}\right)+b(t)\left(u_{t}^{*}-u_{t}\right)\right]
$$

and taking into account equations (0.1) and (2.10), we see that this integral can be written as

$$
-\int_{0}^{T}\left(X_{t}^{*}-X_{t}\right) \mathrm{d} p_{t}-\int_{0}^{T} p_{t} \mathrm{~d}\left(X_{t}^{*}-X_{t}\right)+\int_{0}^{T}\left(X_{t}^{*}-X_{t}\right) \beta_{t} \mathrm{~d} M_{t}^{H} .
$$


Therefore, taking the expectation, since the stochastic integral part gives zero, we get that

$$
\Delta_{2}=\mathbb{E}\left\{q_{T} X_{T}\left[X_{T}^{*}-X_{T}\right]-\int_{0}^{T}\left(X_{t}^{*}-X_{t}\right) \mathrm{d} p_{t}-\int_{0}^{T} p_{t} \mathrm{~d}\left(X_{t}^{*}-X_{t}\right)\right\} .
$$

Now, integrating by parts, since $p_{T}=q_{T} X_{T}$ and $X_{0}^{*}-X_{0}=0$, it comes that $\Delta_{2}=0$ and finally $J\left(u^{*}\right)-J(u)$ $=\Delta_{1} \geq 0$. This of course means that $u$ minimizes $J$ over $\mathcal{U}_{H}$.

Now we show that the minimum value of $J$ over $\mathcal{U}_{H}$ is achieved uniquely and we identify the minimizer:

Lemma 2.4. There exists a unique $u \in \mathcal{U}_{H}$ such that $u$ can be represented as $u=-(b / r) p$ where $(p, \beta)$ is a pair of $\left(\mathcal{F}_{t}^{H}\right)$-adapted processes which satisfies equation (2.10). Moreover for the corresponding pair $(p, \beta)$, the following representations hold:

$$
p_{t}=\pi(t) X_{t}+\int_{0}^{t}\left[\gamma(t, s)-\pi(t) K_{H}^{c}(t, s)\right] \mathrm{d} M_{s}^{H} ; \quad \beta_{t}=\bar{\gamma}(t),
$$

where $\pi, \gamma$ and $\bar{\gamma}$ are defined by equations (0.4, 2.1) and (2.3), respectively.

Proof. At first, we prove the uniqueness. Suppose that $u^{1}$ and $u^{2}$ in $\mathcal{U}_{H}$ both satisfy the required property, i.e., for $i=1,2$, we have $u^{i}=-(b / r) p^{i}$, where $\left(p^{i}, \beta^{i}\right)$ is a pair satisfying equation (2.10) with $X^{i}=X^{u^{i}}$. Let us use the notations

$$
\delta X=X^{1}-X^{2} ; \delta p=p^{1}-p^{2} ; \delta \beta=\beta^{1}-\beta^{2} .
$$

From equations (0.1) and (2.10), we have

$$
\begin{aligned}
\mathrm{d} \delta X_{t} & =a(t) \delta X_{t} \mathrm{~d} t-\frac{b^{2}(t)}{r(t)} \delta p_{t} \mathrm{~d} t, t \in[0, T] ; \delta X_{0}=0, \\
\mathrm{~d} \delta p_{t} & =-a(t) \delta p_{t} \mathrm{~d} t-q(t) \delta X_{t} \mathrm{~d} t+\delta \beta_{t} \mathrm{~d} M_{t}^{H}, t \in[0, T], \delta p_{T}=q_{T} \delta X_{T} .
\end{aligned}
$$

Applying the Itô formula to the product $\delta p_{t} \delta X_{t}$, we obtain immediately that

$$
q_{T}\left[\delta X_{T}\right]^{2}=-\int_{0}^{T}\left\{q(t)\left[\delta X_{t}\right]^{2}+\frac{b^{2}(t)}{r(t)}\left[\delta p_{t}\right]^{2}\right\} \mathrm{d} t+\int_{0}^{T} \delta X_{t} \delta \beta_{t} \mathrm{~d} M_{t}^{H} .
$$

Thus, taking expectation, we get

$$
\mathbb{E} q_{T}\left\{\left[\delta X_{T}\right]^{2}+\int_{0}^{T}\left\{q(t)\left[\delta X_{t}\right]^{2}+\frac{b^{2}(t)}{r(t)}\left[\delta p_{t}\right]^{2}\right\} \mathrm{d} t\right\}=0 .
$$

Consequently $\delta X_{t} \equiv 0, \delta p_{t} \equiv 0$ and hence also $\delta \beta_{t} \equiv 0$. This means in particular that $u^{1} \equiv u^{2}$.

Now we turn to prove the existence. We take

$$
u_{t}=-\frac{b(t)}{r(t)} p_{t} ; \quad p_{t}=\pi(t) \widetilde{X}_{t}+V_{t},
$$

where

$$
\mathrm{d} \widetilde{X}_{t}=a(t)\left[\widetilde{X}_{t}+\widetilde{B}_{t}\right] \mathrm{d} t-\frac{b^{2}(t)}{r(t)}\left[\pi(t) \widetilde{X}_{t}+V_{t}\right] \mathrm{d} t ; \widetilde{X}_{0}=x
$$


with

$$
\widetilde{B}_{t}=\int_{0}^{t} c(s) \mathrm{d} B_{s}^{H} ; \quad V_{t}=\int_{0}^{t} \gamma(t, s) \mathrm{d} M_{s}^{H}
$$

Observe that actually, from (2.13-2.15), it appears that the process $X=\widetilde{X}+\widetilde{B}$ is nothing but the state process $X^{u}$ which corresponds to $u$ by equation (0.1). Moreover, due to the representation (1.5), it is readily seen that we can rewrite $p$ in terms of $X$ in the form given in (2.11).

Now we check that $p$ satisfies the final condition which is required in equation (2.10). Due to the representation (1.5) for $t=T$ and the final condition $\gamma(T, s)=q_{T} K_{H}^{c}(T, s)$ in equation (2.1), from definitions (2.15) we see that $V_{T}=q_{T} \widetilde{B}_{T}$. Consequently, due the final condition $\pi(T)=q_{T}$ in equation (0.4), from definition (2.13) for $t=T$, it is clear that $p_{T}=q_{T} X_{T}$.

Finally, we show that the process $p$ is a semimartingale and we identify its decomposition. Since $\gamma(., s)$ is differentiable, we see that $V$ is a semimartingale and, due to the definition (2.3) of $\bar{\gamma}$, its stochastic differential is

$$
\mathrm{d} V_{t}=\left[\int_{0}^{t} \dot{\gamma}(t, s) \mathrm{d} M_{s}^{H}\right] \mathrm{d} t+\bar{\gamma}(t) \mathrm{d} M_{t}^{H}
$$

Taking the expression of $\dot{\gamma}(., s)$ from the right-hand side of $(2.1)$, this can be rewritten as

$$
\mathrm{d} V_{t}=\left[\int_{0}^{t}\left\{-a(t)\left[\gamma(t, s)+K_{H}^{c}(t, s) \pi(t)\right]-q(t) K_{H}^{c}(t, s)+\frac{b^{2}(t)}{r(t)} \pi(t) \gamma(t, s)\right\} \mathrm{d} M_{s}^{H}\right] \mathrm{d} t+\bar{\gamma}(t) \mathrm{d} M_{t}^{H}
$$

Hence, due to the definition (2.15) of $V$ and $\widetilde{B}$ and taking into account the representation $(1.5)$ of $\widetilde{B}$, we get

$$
\mathrm{d} V_{t}=\left\{-a(t)\left[V_{t}+\pi(t) \widetilde{B}_{t}\right]-q(t) \widetilde{B}_{t}+\frac{b^{2}(t)}{r(t)} \pi(t) V_{t}\right\} \mathrm{d} t+\bar{\gamma}(t) \mathrm{d} M_{t}^{H}
$$

Now, from the definition $(2.13)$ of $p$, we have

$$
\mathrm{d} p_{t}=\dot{\pi}(t) \widetilde{X}_{t} \mathrm{~d} t+\pi(t) \mathrm{d} \widetilde{X}_{t}+\mathrm{d} V_{t}
$$

and, inserting (2.14) and (2.16), we can compute this stochastic differential. Using the equation (0.4) for $\pi$, it is easy to check that actually

$$
\mathrm{d} p_{t}=-a(t) p_{t} \mathrm{~d} t-q(t)\left[\widetilde{X}_{t}+\widetilde{B}_{t}\right] \mathrm{d} t+\bar{\gamma}(t) \mathrm{d} M_{t}^{H}
$$

This means exactly that $\mathrm{d} p_{t}$ is given by the right-hand side of equation (2.10) with $X_{t}=\widetilde{X}_{t}+\widetilde{B}_{t}$ and $\beta_{t}=$ $\bar{\gamma}(t)$. Summarizing, we have checked that the pair $(p, \beta)$ of $\left(\mathcal{F}_{t}^{H}\right)$-adapted processes defined in $(2.11)$ satisfies equation (2.10) with the state process $X$ corresponding to $u=-(b / r) p$.

Now we may turn to the proof of Theorem 2.1.

Proof of Theorem 2.1. Here we use the same notations as in the proof of Lemma 2.4. At first we show that the optimal $u$ in $\mathcal{U}_{H}$ which has just been identified is actually the closed-loop control defined in $(2.5,2.6)$ which then turns to be also optimal in $\mathcal{U}_{\text {ad }}$. We write $p_{t}=\pi(t) X_{t}+v_{t}$ where

$$
v_{t}=V_{t}-\pi(t) \widetilde{B}_{t}
$$

Using the Riccati equation (0.4) for $\pi$ and the equation (2.16) for $V$ to compute the stochastic differential

$$
\mathrm{d} v_{t}=\mathrm{d} V_{t}-\dot{\pi}(t) \widetilde{B}_{t} \mathrm{~d} t-\pi(t) c(t) \mathrm{d} B_{t}^{H}
$$


it is readily seen that

$$
v_{t}=\int_{0}^{t}\left[-a(s)+\frac{b^{2}(s)}{r(s)} \pi(s)\right] v_{s} \mathrm{~d} s+\int_{0}^{t} \bar{\gamma}(s) \mathrm{d} M_{s}^{H}-\int_{0}^{t} \pi(s) c(s) \mathrm{d} B_{s}^{H} .
$$

But, due to the representation (1.7) of a stochastic integral with respect to $M^{H}$ as an integral with respect to $B^{H}$, in terms of the function $\bar{k}$ defined by (2.4), we can rewrite this as

$$
v_{t}=\int_{0}^{t}\left[-a(s)+\frac{b^{2}(s)}{r(s)} \pi(s)\right] v_{s} \mathrm{~d} s+\int_{0}^{t}\left[\frac{\bar{k}(t, s)}{c(s)}-\pi(s)\right] c(s) \mathrm{d} B_{s}^{H} .
$$

Consequently, since due to (0.1) we have $c(s) \mathrm{d} B_{s}^{H}=\mathrm{d} X_{s}-\left[a(s) X_{s}+b(s) u_{s}\right] \mathrm{d} s$, it means that the optimal pair $(u, X)$ is governed by the system $(2.5,2.6)$.

Finally we analyze the optimal cost. Let us recall the decomposition $X=\widetilde{X}+\widetilde{B}$ of the optimal state process and the representation $p=\pi \widetilde{X}+V$ of the corresponding solution of equation (2.10). Using (2.10) and (2.14) and applying the formula of integration by parts to the product $p_{t} \widetilde{X}_{t}$, we can evaluate

$$
\begin{aligned}
p_{T} \widetilde{X}_{T}= & p_{0} \widetilde{X}_{0}+\int_{0}^{T} p_{t} \mathrm{~d} \widetilde{X}_{t}+\int_{0}^{T} \widetilde{X}_{t} \mathrm{~d} p_{t} \\
= & \pi(0) x^{2}+\int_{0}^{T} \widetilde{B}_{t}\left\{[a(t) \pi(t)+q(t)] \widetilde{X}_{t}+a(t) V_{t}+q(t) \widetilde{B}_{t}\right\} \mathrm{d} t \\
& +\int_{0}^{T} \bar{\gamma}(t) \widetilde{X}_{t} \mathrm{~d} M_{t}^{H}-\int_{0}^{T}\left[q(t) X_{t}^{2}+\frac{b^{2}(t)}{r(t)} p_{t}^{2}\right] \mathrm{d} t .
\end{aligned}
$$

Of course when one takes the expectation in both sides the equality remains valid and actually the stochastic integral part in the right-hand side gives zero. Then, observing that

$$
\begin{aligned}
q_{T} X_{T}^{2} & =q_{T} X_{T} \widetilde{X}_{T}+q_{T} X_{T} \widetilde{B}_{T} \\
& =p_{T} \widetilde{X}_{T}+q_{T}\left[\widetilde{X}_{T} \widetilde{B}_{T}+\widetilde{B}_{T}^{2}\right],
\end{aligned}
$$

and that for the optimal control $u$ one has

$$
\frac{b^{2}(t)}{r(t)} p_{t}^{2}=r(t) u_{t}^{2}
$$

from the definition $(0.2)$ of the cost function $J$, we get

$$
J(u)=\pi(0) x^{2}+q_{T}\left[\mathbb{E} \widetilde{X}_{T} \widetilde{B}_{T}+\mathbb{E} \widetilde{B}_{T}^{2}\right]+\mathbb{E} \int_{0}^{T} \widetilde{B}_{t}\left\{[a(t) \pi(t)+q(t)] \widetilde{X}_{t}+a(t) V_{t}+q(t) \widetilde{B}_{t}\right\} \mathrm{d} t .
$$

Recall that $\widetilde{B}_{t}=\int_{0}^{t} K_{H}^{c}(t, s) \mathrm{d} M_{s}^{H}$ and $V_{t}=\int_{0}^{t} \gamma(t, s) \mathrm{d} M_{s}^{H}$. Moreover, due to the equation (2.14), we can also write

$$
\widetilde{X}_{t}=\mathbb{E} \widetilde{X}_{t}+\int_{0}^{t} f(t, s) \mathrm{d} M_{s}^{H}
$$

where for $s \in[0, T]$, the function $f(., s)=(f(t, s), s \leq t \leq T)$ is the solution of the following differential equation in the variable $t$ on $[s, T]$ :

$$
\dot{f}(., s)=\left(a-\frac{b^{2}}{r} \pi\right) f(., s)-\frac{b^{2}}{r} \gamma(., s)+a K_{H}^{c}(., s) ; \quad f(s, s)=0 .
$$


Hence, from (2.18), we obtain that

$$
\begin{aligned}
J(u)= & \pi(0) x^{2}+q_{T} \int_{0}^{T}\left\{f(T, s) K_{H}^{c}(T, s)+\left[K_{H}^{c}(T, s)\right]^{2}\right\} \mathrm{d} w_{s}^{H} \\
& +\int_{0}^{T}[a(t) \pi(t)+q(t)]\left\{\int_{0}^{t} K_{H}^{c}(t, s) f(t, s) \mathrm{d} w_{s}^{H}\right\} \mathrm{d} t \\
& +\int_{0}^{T}\left\{a(t) \int_{0}^{t} K_{H}^{c}(t, s) \gamma(t, s) \mathrm{d} w_{s}^{H}+q(t) \int_{0}^{t}\left[K_{H}^{c}(t, s)\right]^{2} \mathrm{~d} w_{s}^{H}\right\} \mathrm{d} t .
\end{aligned}
$$

Changing the order of integration in the double integrals of the right-hand side, we can rewrite

$$
\begin{aligned}
J(u)= & \pi(0) x^{2}+\int_{0}^{T}\left\{q_{T} f(T, s) K_{H}^{c}(T, s)+\int_{s}^{T} g_{1}(t, s) \mathrm{d} t\right\} \mathrm{d} w_{s}^{H} \\
& +\int_{0}^{T}\left\{q_{T}\left[K_{H}^{c}(T, s)\right]^{2}+\int_{s}^{T} g_{2}(t, s) \mathrm{d} t\right\} \mathrm{d} w_{s}^{H}
\end{aligned}
$$

where

$$
g_{1}(t, s)=[a(t) \pi(t)+q(t)] K_{H}^{c}(t, s) f(t, s) ; \quad g_{2}(t, s)=a(t) K_{H}^{c}(t, s) \gamma(t, s)+q(t)\left[K_{H}^{c}(t, s)\right]^{2} .
$$

But, using equations (2.1) and (2.19), it is easy to check that

$$
q_{T} f(T, s) K_{H}^{c}(T, s)+\int_{s}^{T} g_{1}(t, s) \mathrm{d} t=\int_{s}^{T}\left[a(t) K_{H}^{c}(t, s) \gamma(t, s)-\frac{b^{2}(t)}{r(t)} \gamma^{2}(t, s)\right] \mathrm{d} t .
$$

Inserting this into (2.20) and taking into account the expression (2.2) for the function $\dot{\lambda}(., s)$, we get

$$
J(u)=\pi(0) x^{2}+\int_{0}^{T}\left\{q_{T}\left[K_{H}^{c}(T, s)\right]^{2}-\int_{s}^{T} \dot{\lambda}(t, s) \mathrm{d} t\right\} \mathrm{d} w_{s}^{H} .
$$

Therefore, since the final condition in $(2.2)$ is $\lambda(T, s)=q_{T}\left[K_{H}^{c}(T, s)\right]^{2}$, we see that the following representation of the optimal cost holds:

$$
J(u)=\pi(0) x^{2}+\int_{0}^{T} \lambda(s, s) \mathrm{d} w_{s}^{H} .
$$

Finally, due to the notation $\bar{\lambda}(s)=\lambda(s, s)$ introduced in $(2.3)$, this means that (2.7) holds.

Remark 2.5. Let us justify the observation which is formulated in Remark 2.2. Actually, we can rewrite the component $v_{t}$ defined by $(2.17)$ in the previous proof as

$$
v_{t}=\int_{0}^{t} \mu(t, s) \mathrm{d} M_{s}^{H}
$$

where

$$
\mu(t, s)=\gamma(t, s)-\pi(t) K_{H}^{c}(t, s), s \leq t .
$$

It is readily seen that $\mu$ satisfies the following equation:

$$
\frac{\partial \mu}{\partial t}(t, s)=-\left[a(t)-\frac{b^{2}(t)}{r(t)} \pi(t)\right] \mu(t, s)-\pi(t) \frac{\partial K_{H}^{c}}{\partial t}(t, s) ; \quad \mu(T, s)=0 .
$$


Consequently, in terms of the function $\phi$ defined in (2.9), we have

$$
\mu(t, s)=\int_{t}^{T} \phi(\tau, t) \pi(\tau) \frac{\partial K_{H}^{c}}{\partial \tau}(\tau, s) \mathrm{d} \tau
$$

and

$$
v_{t}=\int_{t}^{T} \phi(\tau, t) \pi(\tau)\left\{\int_{0}^{t} \frac{\partial K_{H}^{c}}{\partial \tau}(\tau, s) \mathrm{d} M_{s}^{H}\right\} \mathrm{d} \tau .
$$

But, due to the representation (1.5) of $\widetilde{B}$, it is clear that for every $\tau \in[t, T]$, the predictor $\hat{B}_{\tau}^{t}=\mathbb{E}\left(\widetilde{B}_{\tau} / X_{s}, s \leq t\right)$ of $\widetilde{B}_{\tau}$ based on the observation of $X$ on $[0, t]$ is given by

$$
\hat{B}_{\tau}^{t}=\int_{0}^{t} K_{H}^{c}(\tau, s) \mathrm{d} M_{s}^{H} ; \quad \frac{\mathrm{d} \hat{B}_{\tau}^{t}}{\mathrm{~d} \tau}=\int_{0}^{t} \frac{\partial K_{H}^{c}}{\partial \tau}(\tau, s) \mathrm{d} M_{s}^{H} .
$$

So, finally, we can represent $v_{t}$ in the form

$$
v_{t}=\int_{t}^{T} \phi(\tau, t) \pi(\tau) \mathrm{d} \hat{B}_{\tau}^{t} .
$$

This means exactly that the equality (2.8) claimed in Remark 2.2 is valid.

Concluding comments. Here we have solved the basic fractional type linear-quadratic Gaussian regulator problem when a complete observation is available. Actually, for a partially observable system where the observation is governed by a linear channel with an independent fractional Brownian noise, we can show that a separation principle holds, i.e., the optimal control separates into two stages based on optimal filtering of the unobservable state and optimal control of the filtered state. This will be reported in a forthcoming paper.

\section{REFERENCES}

[1] M.H.A. Davis, Linear Estimation and Stochastic Control. Chapman and Hall (1977).

[2] L. Decreusefond and A.S. Üstünel, Stochastic analysis of the fractional Brownian motion. Potential Anal. 10 (1999) $177-214$.

[3] T.E. Duncan, Y. Hu and B. Pasik-Duncan, Stochastic calculus for fractional Brownian motion I. Theory. SIAM J. Control Optim. 38 (2000) 582-612.

[4] G. Gripenberg and I. Norros, On the prediction of fractional Brownian motion. J. Appl. Probab. 33 (1997) 400-410.

[5] Y. Hu, B. Øksendal and A. Sulem, A stochastic maximum principle for processes driven by fractional Brownian motion, Preprint 24. Pure Math. Dep. Oslo University (2000).

[6] M.L. Kleptsyna and A. Le Breton, Statistical analysis of the fractional Ornstein-Uhlenbeck type process. Statist. Inference Stochastic Process. (to appear).

[7] M.L. Kleptsyna and A. Le Breton, Extension of the Kalman-Bucy filter to elementary linear systems with fractional Brownian noises. Statist. Inference Stochastic Process. (to appear).

[8] M.L. Kleptsyna, A. Le Breton and M.-C. Roubaud, General approach to filtering with fractional Brownian noises - Application to linear systems. Stochastics and Stochastics Rep. 71 (2000) 119-140.

[9] M.L. Kleptsyna, A. Le Breton and M. Viot, Solution of some linear-quadratic regulator problem under a fractional Brownian perturbation and complete observation, in Prob. Theory and Math. Stat., Proc. of the 8th Vilnius Conference, edited by B. Grigelionis et al., VSP/TEV (to appear).

[10] R.S. Liptser and A.N. Shiryaev, Statistics of Random Processes. Springer-Verlag (1978).

[11] I. Norros, E. Valkeila and J. Virtamo, An elementary approach to a Girsanov formula and other analytical results on fractional Brownian motions. Bernoulli 5 (1999) 571-587.

[12] C.J. Nuzman and H.V. Poor, Linear estimation of self-similar processes via Lamperti's transformation. J. Appl. Probab. 37 (2000) 429-452. 